\title{
Polymorphisms in the promoter region of catalase gene and essential hypertension
}

\author{
Xiao Feng Zhou ${ }^{\mathrm{a}, * *}$, Jing Cui ${ }^{\mathrm{b}}$, Anita L. DeStefano ${ }^{\mathrm{d}, \mathrm{e}}$, Irmarie Chazaro ${ }^{\mathrm{b}, \mathrm{e}}$, Lindsay A. Farrer ${ }^{\mathrm{c}, \mathrm{d}, \mathrm{f}}$, \\ Athanasios J. Manolis ${ }^{\mathrm{g}}$, Haralambos Gavras ${ }^{\mathrm{b}}$ and Clinton T. Baldwin ${ }^{\mathrm{a}, *}$ \\ ${ }^{a}$ Center for Human Genetics, Department of Medicine, Boston University School of Medicine, Boston, MA, USA \\ ${ }^{\mathrm{b}}$ Hypertension Section, Boston University School of Medicine, Boston, MA, USA \\ ${ }^{\mathrm{c}}$ Genetics Program, Boston University School of Medicine, Boston, MA, USA \\ ${ }^{\mathrm{d}}$ Department of Neurology, Boston University School of Medicine, Boston, MA, USA \\ e Department of Biostatistics, Boston University School of Public Health, Boston, MA, USA \\ ${ }^{\mathrm{f}}$ Department of Epidemiology, Boston University School of Public Health, Boston, MA, USA \\ ${ }^{\mathrm{g}}$ Cardiology Division, Tzanion Hospital, Piraeus, Greece
}

\begin{abstract}
Genetic variations that predispose individuals to complex disorders, such as essential hypertension, may be found in gene coding regions, intronic regions or in gene promoter regions. Most studies have focused on gene variations that result in amino acid substitutions because they result in different isoforms of the protein, presumably resulting in differences in protein properties. Less attention has been placed on the role of intronic or promoter mutations. In this report, we examined two single nucleotide polymorphisms (SNPs) in the catalase (CAT) gene prompter region in a cohort of hypertensive Caucasians and African Americans with a Mass Spec based Homogenous MassEXTEND assay. We found an association when a specific combination of the two promoter SNPs was examined in Caucasians. No association was observed in African Americans. Our data suggest that genetic variations in the promoter region of catalase gene influence the susceptibility to essential hypertension. In addition, the genetic factors that contribute to hypertension maybe different between ethnic groups.
\end{abstract}

Keywords: Catalase, essential hypertension, SNP, promoter

\section{Introduction}

Essential hypertension is an idiopathic elevation of blood pressure. It affects approximately $24 \%$ of the adult population in US [1] and is a recognized risk factor for cardiovascular, cerebrovascular and renal disease. Although the etiology of essential hypertension is unknown, it involves the interaction between heritable and environmental factors. The major neurohumoral mechanisms involved in systemic blood pres-

\footnotetext{
* Corresponding author: Clinton T. Baldwin, $\mathrm{PhD}$, Center for $\mathrm{Hu}-$ man Genetics, Boston University School of Medicine, 715 Albany Street, W408, Boston, MA 02118, USA. Tel.: +1 617638 7086; Fax: +1 617638 7092; E-mail: cbaldwin@bu.edu.

${ }^{* *}$ Present address: Dental Research Institute, School of Dentistry, University of California at Los Angeles, Los Angeles, CA, USA.
}

sure regulation and hypertensive complications are well understood and include the renin-angiotensin aldosterone system [2], the sympathoadrenal system [3], the kallikrein-kinin system [4] and others. There are also numerous enzymes such as catalase [5] affecting the metabolism of local tissue protective or damaging factors [6], which have been described to contribute to blood pressure dysregulation. The genes for these enzymes are likely candidates for molecular genetic analysis, because their variants may predispose individuals to hypertension.

Numerous studies have demonstrated association between hypertension and polymorphisms of genes such as angiotensin converting enzyme [7], angiotensinogen $[8,9]$, beta2-adrenergic receptor [10], and alphaadducin $[11,12]$ in some hypertensive populations. Fewer reports have examined the association between 
promoter mutations and hypertension. Two single nucleotide polymorphisms (SNPs) have been reported in the Catalase gene (CAT), one (CAT-844) being associated with higher blood pressure among a group of Chinese hypertensives [13] and the second (CAT-262) showing association with catalase activity levels in a Swedish population [14]. Catalase regulates the plasma level of reactive oxygen species [5] and together with nitric oxide (NO), it influences angiotensin converting enzyme activation, LDL oxidation, adhesion molecule expression, platelet aggregation, endothelial cell apoptosis, and vascular smooth cell growth [6].

In this report, we examined these CAT SNPs in a collection of hypertensive Caucasians and African Americans and ethnically matched normotensive controls and found an association with hypertension. Our results suggest that promoter mutations in CAT gene may contribute to the development of essential hypertension.

\section{Materials and methods}

\subsection{Study population}

African American patients with essential hypertension were identified from several clinics at the Boston Medical Center, Boston MA USA. Caucasian patients were identified at Tzanion Hospital, Piraeus Greece. All subjects provided informed consent and human subject review boards at both institutions approved this study. Individuals were classified as hypertensive if pretreatment systolic blood pressure was $\geqslant 140 \mathrm{~mm} \mathrm{Hg}$, pretreatment diastolic blood pressure was $\geqslant 90 \mathrm{~mm} \mathrm{Hg}$, or the individual was being treated for hypertension. A routine clinical evaluation was carried out and, whenever clinically appropriate, additional standard diagnostic procedures were conducted to exclude organic causes of secondary hypertension (eg, renovascular lesions, adrenal tumors). Ethnically matched normotensive subjects older than 55 years were recruited from the same populations from which the hypertensive individuals were ascertained. Given available sample size, to explore candidate gene association study using casecontrol, assuming the high risk allele frequency is 0.3 , and prevalence for hypertension is 0.3 , and type I error chose 0.05 , for all the markers linked to the disease locus at $\mathrm{LD}\left(D^{\prime}=0.8\right)$ and allele frequency around 0.3 , we will have 0.86 power to detect the association (http://statgen.iop.kcl.ac.uk/gpc/cc2.html).

\subsection{Genotyping}

The genomic DNA was extracted from peripheral blood using Puregene kit (Gentra Systems, Inc). The SNP genotypes were determined with the Homogenous MassEXTEND assay (Sequenom, San Diego, CA) according to the manufacture's instruction, with minor modification. The primers used for experimentation are shown in Table 1. After PCR amplification of the target regions, non-incorporated dNTPs were removed by shrimp alkaline phosphatase. A detecting primer immediately upstream from the polymorphic site was added together with a specific combination of deoxy and di-deoxy dNTPs and Thermosequanase (Amersham). The extension reaction was performed according to the manufacturer's protocol $\left(94^{\circ} \mathrm{C} 2 \mathrm{~min}\right.$, followed by 80 cycles of $94^{\circ} \mathrm{C} 5 \mathrm{sec}, 52^{\circ} \mathrm{C} 5 \mathrm{sec}, 72^{\circ} \mathrm{C}$ $5 \mathrm{sec})$. The extension products were then analyzed by mass spectrometry (Sequenom, San Diego, CA).

\subsection{Statistical analysis}

Differences between cases and controls were assessed using t-tests (age, body mass index, age at onset of hypertension) or chi square tests (kidney disease (yes/no), diabetes (yes/no), coronary heart disease (yes/no) and stroke (yes/no)). Allele and genotype frequencies were compared between hypertensive and control individuals for each ethnic group by a $\chi^{2}$ test.

To explore linkage disequilbrium and to predict haplotypes for the two SNPs in the catalase promoter we used Arlequin 2.0 (http://lgb.unige.ch/arlequin/). We obtained maximum likelihood estimates of haplotype frequencies by implementing the expectationmaximization (EM) algorithm in Arlequin 2.0. These haplotype frequencies were used to compute a standardized measure of linkage disequilibrium coefficient, D'. The haplotype frequency distributions were compared between hypertensive and normotensive groups.

Specific combinations of genotypes for the two SNPs in the catalase were assessed using logistic regression. Different genetic models were explored. For example, we assumed a recessive mode of inheritance for CAT844 and a dominant mode of inheritance for CAT-262. In this model individuals with genotype AA at CAT844 and with at least one T at CAT-844 were hypothesized to have increase odds of hypertension. The reference group in this case would be individuals who are not homozygous (AA) at the first SNP but who are homozygous (CC) at the second SNP. Other models (e.g. recessive mode of inheritance at both SNPs, dominant 
Table 1

Homogenous MassEXTEND assay for SNP genotyping

\begin{tabular}{|c|c|c|c|}
\hline SNPs & Extension Nucleotides & \multicolumn{2}{|c|}{ Products } \\
\hline $\begin{array}{l}\text { CAT-844 } \\
\text { Amplification primers } \\
5^{\prime} \text { TTAAAGACACAAAGCTTTTCAAAATTCC3' } \\
5^{\prime} \text { GGGAAAAATGTATTCAACTCTGGTACAC3' } \\
\text { Detecting primer } \\
5^{\prime} \text { CAAAATTCCTGCTTACCTGGG3' (6381.2Da) }\end{array}$ & $\begin{array}{l}\text { dGTP, } \\
\text { ddATP, ddCTP, ddTTP }\end{array}$ & $\begin{array}{l}\text { A allele } \\
+ \text { ddA }(6678.4 \mathrm{Da})\end{array}$ & $\begin{array}{l}\text { G allele } \\
+ \text { GGddT }(7327.8 \mathrm{Da})\end{array}$ \\
\hline $\begin{array}{l}\text { CAT-262 } \\
\text { Amplification primers } \\
5^{\prime} \text { GGCCTGAAGGATGCTGATAACCGGGAGC3' } \\
5^{\prime} \text { ATACCCAGGAGGGTGCGGAAAGGAAGGG3' } \\
\text { Detecting primer } \\
5^{\prime} \text { CGCCCTGGGTTCGGCTAT3' }^{\prime}(5482.6 \mathrm{Da})\end{array}$ & $\begin{array}{l}\text { dTTP, } \\
\text { ddATP, ddCTP, ddGTP }\end{array}$ & $\begin{array}{l}\text { C allele } \\
+ \text { ddC }(5755.8 \mathrm{Da})\end{array}$ & $\begin{array}{l}\text { T allele } \\
+ \text { TddC (6060Da) }\end{array}$ \\
\hline
\end{tabular}

Table 2

Clinical characterization of the study population

\begin{tabular}{lll}
\hline & Control subjects & Hypertensive subjects \\
\hline Caucasian & $N=93$ & $N=100$ \\
Gender (male\%) & 63.4 & 58 \\
Recruiting age, y ** & $72.5+8.0$ & $57.7+10.3$ \\
Age at which hypertension diagnosed, y & & $48.2+10.1$ \\
BMI, kg/m ${ }^{2} * *$ & $25.4+4.5$ & $27.3+3.9$ \\
Kidney disease, \% & 0 & 3.0 \\
Diabetes, \% & 12.9 & 11.0 \\
Coronary heart disease, \% & 14.0 & 10.0 \\
Stroke, \% & 4.3 & 3.0 \\
African American & $N=98$ & $N=120$ \\
Gender (male\%) & 32.7 & 37.5 \\
Recruiting age, y & $63.2+7.1$ & $61.8+12.6$ \\
Age at which hypertension diagnosed, y & & $42.7+14.0$ \\
BMI, kg/m ${ }^{2}$ & $29.9+6.3$ & $31.6+7.6$ \\
Kidney disease, $\% *$ & 2.1 & 10.1 \\
Diabetes, \%** & 14.6 & 30.8 \\
Coronary heart disease, \% & 3.1 & 6.7 \\
Stroke, \% ** & 0 & 9.2 \\
\hline
\end{tabular}

Values are mean $+\mathrm{SD} .{ }^{*} p<0.05 ;{ }^{* *} p<0.01$.

mode of inheritance at both SNPs) were evaluated by altering the coding scheme to define the appropriate comparison groups. All logistic regression analyses were conducted using SAS v8.2.

\section{Results}

\subsection{Study population}

Clinical information was collected from 100 hypertensive and 93 normotensive Caucasian subjects and 120 hypertensive and 98 normotensive African American subjects (Table 2). The normotensive and hypertensive groups from each population were of similar age. Caucasian hypertensive subjects had a higher mean BMI than the their normotensive counterparts $(p<0.01)$ but there was no difference in the frequency of kidney disease, diabetes, coronary heart disease. In contrast, African American hypertensive subjects had a significantly higher frequency of diabetes $(p<0.01)$, stroke $(p<0.01)$ and kidney disease $(p<0.05)$ than normotensive controls.

\subsection{Association studies with the CAT-844 and CAT-262 SNPS}

Shown in Table 3 are the allele and genotype frequencies for the two CAT SNP's in the hypertensive and normotensive groups. No association between hypertension and CAT-844 ( $p=0.81$ for Caucasians and 0.40 for African Americans) or with CAT-262 ( $p=0.43$ for Caucasians and 0.39 for African Americans) in either population was observed. Similarly, no association 
Table 3

Allelic and genotypic frequencies among hypertensives and control subjects

\begin{tabular}{cccccc}
\hline & \multicolumn{2}{c}{ Caucasian } & & \multicolumn{2}{c}{ African American } \\
\cline { 2 - 3 } \cline { 6 - 6 } & Control subjects & Hypertensive subjects & & Control subjects & Hypertensive subjects \\
\hline CAT-844 & & & & \\
f(AA) & $34(0.374)$ & $43(0.457)$ & & $34(0.358)$ & $36(0.379)$ \\
f(AG) & $47(0.517)$ & $35(0.372)$ & & $48(0.505)$ & $36(0.379)$ \\
f(GG) & $10(0.110)$ & $16(0.170)$ & & $13(0.137)$ & $23(0.242)$ \\
p & & 0.13 & & & 0.10 \\
f(G) & $67(0.368)$ & $67(0.356)$ & & $74(0.389)$ & $82(0.432)$ \\
p & & 0.81 & & & 0.40 \\
CAT-262 & & & & & \\
f(TT) & $56(0.622)$ & $56(0.596)$ & & $35(0.875)$ & $30(0.938)$ \\
f(TC) & $32(0.356)$ & $32(0.34)$ & & $5(0.125)$ & $2(0.062)$ \\
f(CC) & $2(0.022)$ & $6(0.064)$ & & - & - \\
p & & 0.38 & & & 0.37 \\
f(T) & $36(0.2)$ & $44(0.234)$ & & $5(0.063)$ & $2(0.031)$ \\
p & & 0.43 & &
\end{tabular}

Values in parentheses are frequencies.

Table 4

Predicted haplotype frequencies of CAT-844 and CAT-262 for Caucasians

\begin{tabular}{cccc}
\hline & & Control subjects & Hypertensive subjects \\
\hline-844 & -262 & & \\
A & C & 0.427 & 0.397 \\
A & T & 0.202 & 0.239 \\
G & C & 0.371 & 0.364 \\
\hline
\end{tabular}

was observed when comparing the genotype frequency $(p=0.13$ and 0.10 for CAT-844 and $p=0.38$ and 0.37 for CAT-262 for Caucasian and African American, respectively).

Table 3 also highlights ethnic differences among the frequencies of these alleles. The allele frequency of CAT-844 in the Caucasian (G: 36.2\%) and African American population (G: $41.1 \%$ ) were similar to each other but differs from that reported ${ }^{15}$ for the Chinese hypertensive cohort (G: 68.4\%). The frequency of the CAT-262 SNP in the Caucasian population (T: $21.7 \%$ ) was similar to that reported ${ }^{16}$ for a Swedish population (T: 28.7\%). African American population contained predominantly $\mathrm{C}$ alleles $(95.1 \%)$. In Caucasians, the two SNPs, CAT-262 and CAT-844, were in linkage disequilibrium $\left(r^{2}=0.146\right)$.

Haplotype analysis using the catalase SNPs was performed only in the Caucasian population because of the low frequency of the CAT-262 allele in African Americans. Given the disequilbrium between the two SNP's only three haplotypes are predicted (Table 4). There was no difference between the estimated haplotype frequencies in hypertensives and normotensives ( $p=0.68)$ indicating no association with hypertension.

A logistic regression test was performed to evaluate genotype combinations (dominant-dominant, domin-
Table 5

Odd ratios for hypertension according to genotype combination

\begin{tabular}{|c|c|c|c|}
\hline \multicolumn{2}{|c|}{ Model } & \multirow{2}{*}{$\mathrm{OR}(95 \% \mathrm{CI})$} & \multirow{2}{*}{$\mathrm{p}$-value } \\
\hline CAT-844 & CAT-262 & & \\
\hline AA & $\mathrm{T}_{-}$ & $2.158(1.060-4.393)$ & 0.0339 \\
\hline AA & TT & $3.035(0.569-15.456)$ & 0.1813 \\
\hline A_ & $\mathrm{T}_{\mathbf{Z}}$ & $1.138(0.627-2.065)$ & 0.6701 \\
\hline A_ & TT & $3.035(0.569-15.456)$ & 0.1813 \\
\hline
\end{tabular}

ant-recessive, recessive-dominant, recessive-recessive) between the two SNPs. There was an increased odds for hypertension $(\mathrm{OR}=2.158, p=0.0339)$ when assessing a recessive effect for CAT-844 (AA) and dominant effect for the CAT-262 (T). An increased odds for hypertension was also observed when fitting a recessive effect for both SNPs, CAT-844 (AA) and CAT-262 (TT). This increase was not statistically significant (OR $=3.035, p=0.18$ ), however, likely due to the small number of individuals that were homozygous for the alleles specified in this model.

\section{Discussion}

In this report, we assessed the association between SNPs in the promoter region of the CAT genes and essential hypertension in Greek Caucasians and African Americans. An association was found with the specific genotype combination of CAT-844 homozygous AA together with CAT-262 CT or TT. This association was found in Caucasians only. However, the observation between catalase and hypertension in Caucasians appears to be complex and not evident when individual allele frequency or haplotypes were examined. This may be due to lack of power to detect complex interactions between SNPs. 
An association between CAT and hypertension is consistent with a study in China showing association of individuals homozygous for CAT-844 AA and elevated blood pressure [13]. However, the CAT-844 G allele is more frequent in this Chinese population $(68.4 \%)$ than the two groups reported here (41.1\% for African Americans and $36.2 \%$ for Caucasians). This may be due to population differences or selection bias in the Chinese sample which included hypertensive subjects only. The CAT-262 SNP has not been previously examined with respect to blood pressure values, although the CAT-262 $\mathrm{T}$ allele is associated with higher expression level of the catalase gene [14].

The CAT-844 SNP lies within several predicted transcription factor recognition sites in the promoter region. The $\mathrm{G}$ allele contains putative MZF1 and AP2 binding sites, whereas the A allele contains putative Ikaros-2 and LYK-1 binding sites [13]. In addition, the CAT-262 SNP lies near putative cis-acting sequences for AP-1 and Sp-1 and each form of the SNP has been shown to result in a different nuclear protein binding patterns as well as differences in transcriptional activity [14].

In conclusion, our data suggest that genetic variations in the promoter region of catalase gene influence the susceptibility to essential hypertension. Our results suggest that individual SNPs may not be as important as the specific interaction among several SNPs. We also find that the genetic factors that contribute to hypertension are likely to be different between ethnic groups. Further studies of SNPs in a large number genes involved in blood pressure regulation will be required to identify the full array of genetic factors that cause hypertension.

\section{Acknowledgments}

This work was supported in part by a NIH, grant HL55001 and a NIH training grant HL07224 (to X. Zhou).

\section{References}

[1] V.L. Burt, P. Whelton, E.J. Roccella, C. Brown, J.A. Cutler, M. Higgins, M.J. Horan and D. Labarthe, Prevalence of hypertension in the US adult population. Results from the Third Na- tional Health and Nutrition Examination Survey, 1988-1991, Hypertension 25(3) (1995), 305-313.

[2] H. Gavras, H.R. Brunner and J.H. Laragh, Renin and aldosterone and the pathogenesis of hypertensive vascular damage, Prog Cardiovasc Dis 17(1) (1974), 39-49.

[3] D.S. Goldstein, Plasma norepinephrine in essential hypertension. A study of the studies, Hypertension 3(1) (1981), 48-52.

[4] O.A. Carretero and A.G. Scicli, Local hormonal factors (intracrine, autocrine, and paracrine) in hypertension, Hypertension 18(3) (1991), I58-69.

[5] A. Deisseroth and A.L. Dounce, Catalase: Physical and chemical properties, mechanism of catalysis, and physiological role, Physiol Rev 50(3) (1970), 319-375.

[6] U. Landmesser and D.G. Harrison, Oxidative stress and vascular damage in hypertension, Coron Artery Dis 12(6) (2001), $455-461$.

[7] C.J. O’Donnell, K. Lindpaintner, M.G. Larson, V.S. Rao, J.M. Ordovas, E.J. Schaefer, R.H. Myers and D. Levy, Evidence for association and genetic linkage of the angiotensin-converting enzyme locus with hypertension and blood pressure in men but not women in the Framingham heart study, Circulation 97 (1998), 1766-1772.

[8] X. Jeunemaitre, F. Soubrier, Y.V. Kotelevtsev, R.P. Lifton, C.S. Williams, A. Charru, S.C. Hunt, P.N. Hopkins, R.R. Williams, J.M. Lalouel and P. Corvol, Molecular basis of human hypertension: role of angiotensinogen, Cell 71 (1992), 169-180

[9] H. Iso, S. Harada, T. Shimamoto, S. Sato, A. Kitamura, T. Sankai, T. Tanigawa, M. Iidaa and Y. Komachi, Angiotensinogen T174M and M235T variants, sodium intake and hypertension among non-drinking, lean Japanese men and women, J Hypertens 18(9) (2000), 1197-1206.

[10] K. Ranade, W.H. Shue, Y.J. Hung, C.A. Hsuing, F.T. Chiang, R. Pesich, J. Hebert, M. Olivier, Y.D. Chen, R. Pratt, R. Olshen, D. Curb, D. Botstein, N. Risch and D.R. Cox, The glycine allele of a glycine/arginine polymorphism in the beta2-adrenergic receptor gene is associated with essential hypertension in a population of Chinese origin, Am J Hypertens 14(12) (2001), 1196-1200.

[11] M.A. Province, D.K. Arnett, S.C. Hunt, C. LeiendeckerFoster, J.H. Eckfeldt, A. Oberman, R.C. Ellison, G. Heiss, S.C. Mockrin and R.R. Williams, Association between the alpha-adducin gene and hypertension in the HyperGEN Study, Am J Hypertens 13(6) (2000), 710-718.

[12] O. Melander, K. Bengtsson, M. Orho-Melander, U. Lindblad, C. Forsblom, L. Rastam, L. Groop and U.L. Hulthen, Role of the Gly460Trp polymorphism of the alpha-adducin gene in primary hypertension in Scandinavians, J Hum Hypertens 14(1) (2000), 43-46.

[13] Z. Jiang, J.M. Akey, J. Shi, M. Xiong, Y. Wang, Y. Shen, X. Xu, H. Chen, H. Wu, J. Xiao, D. Lu, W. Huang and L. Jin, A polymorphism in the promoter region of catalase is associated with blood pressure levels, Hum Genet 109(1) (2001), 95-98.

[14] L. Forsberg, L. Lyrenas, U. de Faire and R. Morgenstern, A common functional C-T substitution polymorphism in the promoter region of the human catalase gene influences transcription factor binding, reporter gene transcription and is correlated to blood catalase levels, Free Radic Biol Med 30(5) (2001), 500-505. 


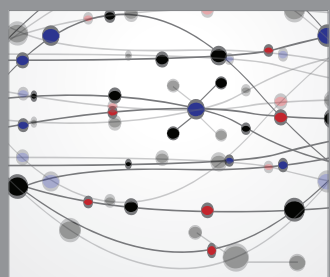

The Scientific World Journal
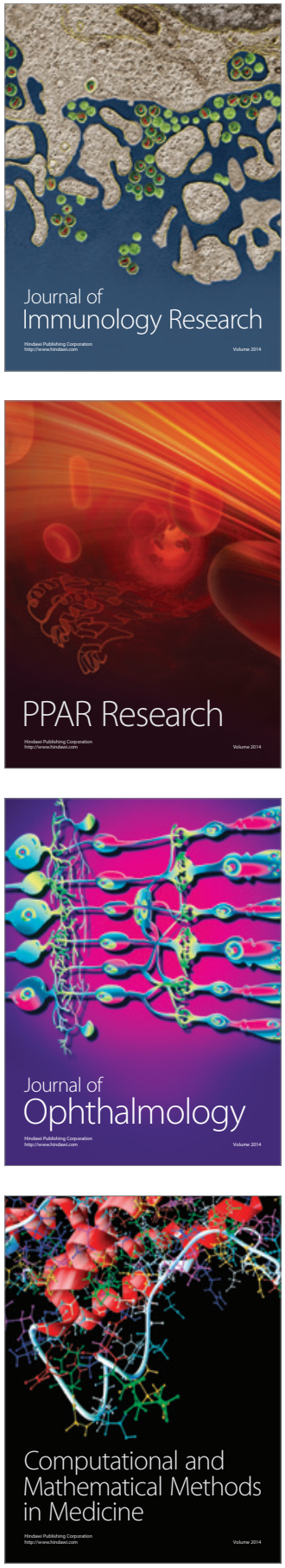

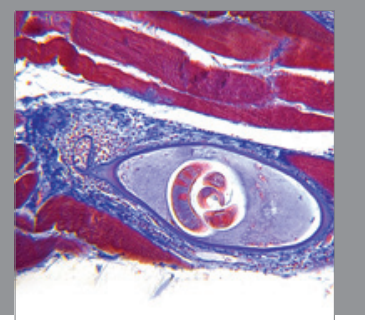

Gastroenterology

Research and Practice
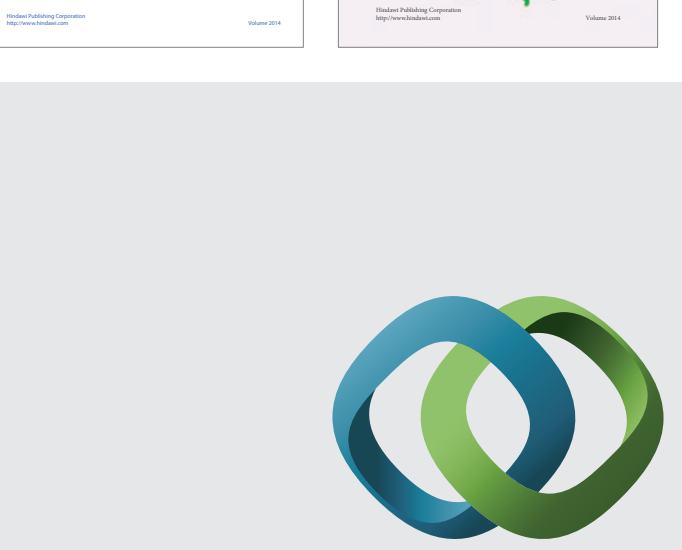

\section{Hindawi}

Submit your manuscripts at

http://www.hindawi.com
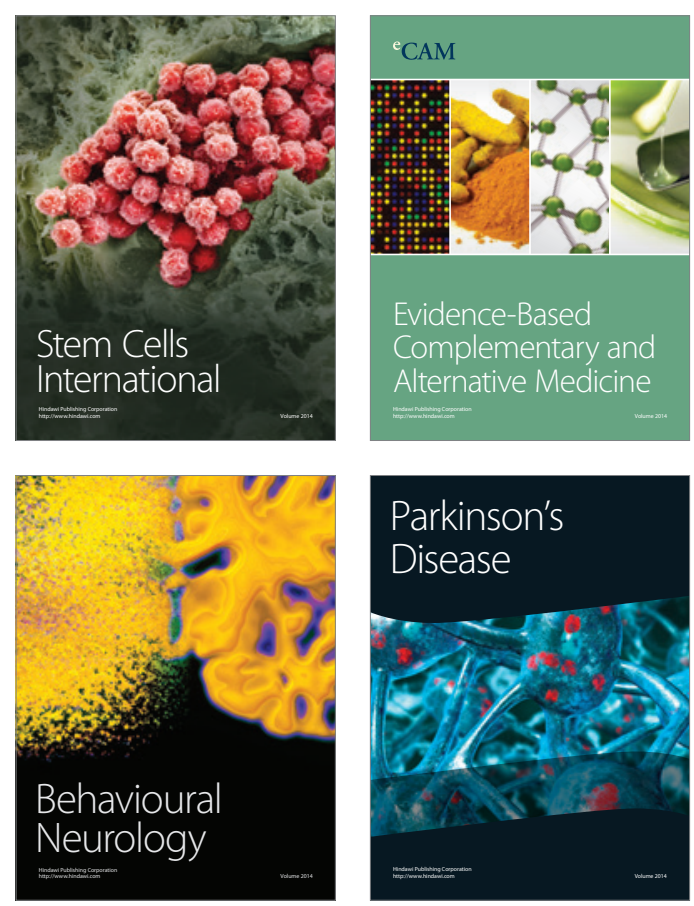

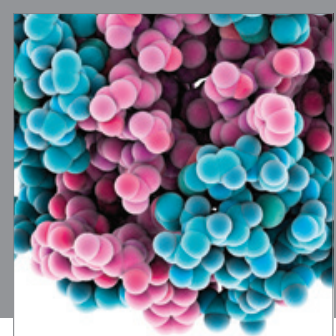

Journal of
Diabetes Research

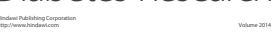

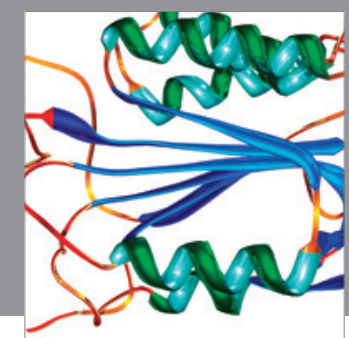

Disease Markers
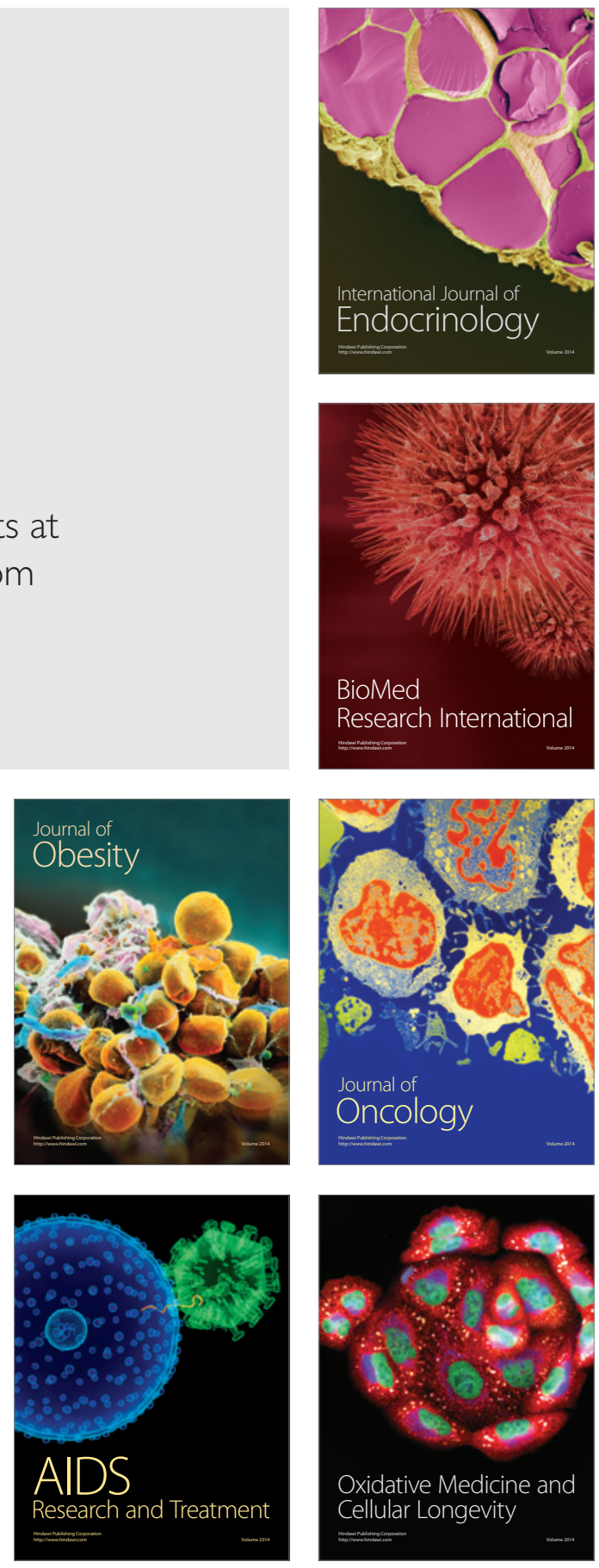